\title{
Genetic Susceptibility to Dental Caries on Pit and Fissure and Smooth Surfaces
}

\author{
J.R. Shaffer ${ }^{a} \quad$ X. Wang ${ }^{b-d, i} \quad$ R.S. DeSensi ${ }^{b-d, ~ i ~}$ \\ K.T. Cuenco ${ }^{b-e, i} \quad$ R. Crout ${ }^{c, g, i} \quad$ D.W. McNeil ${ }^{\text {h }}$ \\ S. Wendell ${ }^{b-d, i}$ \\ R.J. Weyant ${ }^{c, e, ~ i ~}$ \\ M.L. Marazita ${ }^{a-d, f}$ \\ ${ }^{a}$ Department of Human Genetics, Graduate School of Public Health, ${ }^{b}$ Center for Craniofacial and Dental \\ Genetics, School of Dental Medicine, 'Center for Oral Health Research in Appalachia, University of Pittsburgh, \\ Pittsburgh, Pa. and West Virginia University, Morgantown, W.Va., ${ }^{d}$ Department of Oral Biology, School of Dental \\ Medicine, ' Department of Dental Public Health and Information Management, School of Dental Medicine, and \\ ${ }^{f}$ Clinical and Translational Science Institute, and Department of Psychiatry, School of Medicine, University of

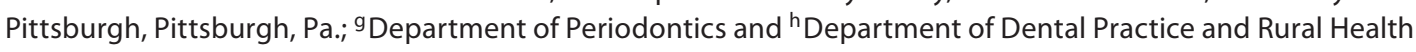 \\ and Department of Psychology, West Virgina University, Morgantown, W.Va., USA
}

\section{Key Words}

Dental caries • Genetic correlation • Genetics • Heritability •

Permanent dentition • Pit and fissure surfaces · Primary

dentition $\cdot$ Smooth surfaces

\begin{abstract}
Carious lesions are distributed nonuniformly across tooth surfaces of the complete dentition, suggesting that the effects of risk factors may be surface-specific. Whether genes differentially affect caries risk across tooth surfaces is unknown. We investigated the role of genetics on two classes of tooth surfaces, pit and fissure surfaces (PFS) and smooth surfaces (SMS), in more than 2,600 subjects from 740 families. Participants were examined for surface-level evidence of dental caries, and caries scores for permanent and/or primary teeth were generated separately for PFS and SMS. Heritability estimates $\left(h^{2}\right.$, i.e. the proportion of trait variation due to genes) of PFS and SMS caries scores were obtained using likelihood methods. The genetic correlations between PFS and SMS caries scores were calculated to assess the degree to which traits covary due to common genetic effects. Overall, the heritability of caries scores was similar for PFS $\left(h^{2}=19-53 \% ; p<0.001\right)$ and SMS $\left(h^{2}=17-42 \% ; p<0.001\right)$.
\end{abstract}

Heritability of caries scores for both PFS and SMS in the primary dentition was greater than in the permanent dentition and total dentition. With one exception, the genetic correlation between PFS and SMS caries scores was not significantly different from 100\%, indicating that (mostly) common genes are involved in the risk of caries for both surface types. Genetic correlation for the primary dentition dfs (decay + filled surfaces) was significantly less than $100 \%(p<0.001)$, indicating that genetic factors may exert differential effects on caries risk in PFS versus SMS in the primary dentition.

Copyright $\odot 2012$ S. Karger AG, Basel

Dental caries is a multifactorial disease widely acknowledged to be caused by a combination of environmental and behavioral factors and genetic predispositions. The environmental risk factors for dental caries have been studied for decades, and include dietary behaviors, bacterial flora, transmission of bacteria among hosts, hygiene, salivary composition and flow rate, tooth positional and morphological features, and fluoride ex-

J.R.S. and X.W. are co-first authors.

\section{KARGER}

Fax +41613061234 E-Mail karger@karger.ch www.karger.com
(C) 2012 S. Karger AG, Basel

0008-6568/12/0461-0038\$38.00/0

Accessible online at:

www.karger.com/cre
Mary L. Marazita, PhD

Center for Craniofacial and Dental Genetics

University of Pittsburgh, Suite 500 Bridgeside Point, 100 Technology Dr

Pittsburgh, PA 15219 (USA)

Tel. +1 412648 8380, E-Mail marazita@ pitt.edu 
posure, socioeconomic status, and access to oral health care [Hunter, 1988; Anderson, 2002; Law et al., 2007; Mobley et al., 2009]. The role of genetics, which continues to be an active area of research, has been investigated in several recent studies [Goodman et al., 1959; Boraas et al., 1988; Liu et al., 1998; Shuler, 2001; Bretz et al., 2005a, b, 2006; Vieira et al., 2008; Wang et al., 2010; Wendell et al., 2010]. Adding to the complexity of dental caries etiology is the nonuniform risk of caries across tooth surfaces of the complete dentition. The sequence and pattern of carious lesions demonstrates a surface hierarchy in susceptibility to decay, with pit and fissure surfaces (PFS) exhibiting much greater risk than smooth surfaces (SMS) [Batchelor and Sheiham, 2004; Psoter et al., 2009].

According to conventional wisdom, the disparity in caries risk between SMS and PFS may partly be explained by differential effects of environmental and behavioral exposures. Notable examples are the benefits of sealants on PFS and fluoride on SMS. For example, professionally administered fluoride treatments have been shown to reduce incidence of caries on the SMS, but not PFS, of the first permanent molar [Jiang et al., 2005]. Additional studies have shown a greater effect of fluoridated water on SMS caries progression, and that snack food and socioeconomic factors affect PFS caries progression [Maupome et al., 2001]. Likewise, a longitudinal study reported that tooth brushing frequency and powered beverage consumption were significantly associated with the progression of childhood caries for PFS (but not SMS), and that noncavitated lesions were more likely to progress to cavitated lesions with age for PFS than for SMS [Warren et al., 2006]. Though few studies have rigorously explored the differential effects of environmental factors on PFS and SMS caries, several risk factors have been observed exhibiting important differences in effects across surface types. Whether SMS caries risk and PFS caries risk are also differentially affected by genetic factors is currently unknown.

To address this question, we examined the role of genetic factors on dental caries separately for SMS and PFS as part of ongoing efforts by the Center for Oral Health Research in Appalachia (COHRA), a partnership between the University of Pittsburgh and West Virginia University. We hypothesized that both SMS and PFS are under genetic control (i.e. heritable), and that the genes involved in caries risk may differ between SMS and PFS. We tested this hypothesis by estimating the heritability (i.e. proportion of variation explained by genetics) of caries scores in primary and permanent dentitions separate- ly for SMS and PFS, and modeling the degree to which variation in caries scores was attributable to common (or different) genetic factors for SMS and PFS.

\section{Subjects and Methods}

\section{Recruitment and Data Collection}

The principal goal of COHRA was to identify the community-, family- and individual-level factors related to oral diseases in the Appalachian population [Polk et al., 2008], a group with notably poor oral health in comparison to the greater US population [Grembowski et al., 1987; Purnell and Counts, 1998; From the Centers for Disease Control and Prevention, 1999; Janes et al., 1999; Public Health and Aging, 2003]. Household-based recruitment of COHRA participants was carried out in Allegheny, Washington and McKean counties of Pennsylvania, and Webster and Nicholas counties of West Virginia. To meet eligibility criteria, households were required to contain one biological parentoffspring pair with the child being 1-18 years of age. All recruitment was conducted without regard to participants' oral health status, and all members of eligible households were invited into the study without regard to biological and/or legal relationships. Participants were excluded according to the following criteria: neurological impairment, severe physical or mental disability, and psychosis. Households were excluded if one member of the biological parent-offspring pair suffered from diminished capacity to resist infection or from blood clots. Written informed consent was obtained from all adult participants. Assent with parentor guardian-written consent was obtained for all child participants. All forms and procedures were approved by the COHRA research committee and the Institutional Review Boards of the University of Pittsburgh and West Virginia University.

Our population-based household recruitment methods yielded a sample that accurately reflects the Appalachian population, including households from a range of socioeconomic statuses. However, as with the general Appalachian population, the majority of households recruited in this study are low-income (i.e. the median annual household income was less than \$25,000), indicating that some oral health risk factors may be more prevalent in this population compared to the greater US, although we believe the effects of such risk factors may be largely generalizable.

732 households comprising a total of 2,663 individuals from 740 biological kinships were enrolled. Families consisting of confirmed biological relatives ranged from 1 to 20 individuals (mean $=4.72$ members). In total, 3,232 relative pairs were available for analysis, which included 1,817 parent-offspring pairs, 756 sibling pairs, 347 half-sibling pairs, 120 avuncular (i.e. uncle/ aunt-nephew/niece) pairs, 104 first-cousin pairs, and 88 other relative pairs. The accuracy of reported familial relationships was validated using panels of ancestry-informative [Marosy et al., 2007] and whole-genome [Cornelis et al., 2010] genetic marker data genotyped by the Center for Inherited Disease Research at Johns Hopkins University and quality checked jointly with the Coordinating Center for the NIH Genes and Environment Initiative (GENEVA) [Laurie et al., 2010]. Standard relationship-testing methods [O'Connell and Weeks, 1998] were used to identify and correct relationship errors prior to conducting the analysis reported herein. 
Dental caries were assessed during dental exams conducted by dentists or research dental hygienists (the latter being calibrated to the reference dentist at least once per year). Inter- and intraexaminer concordance of caries assessments was high [Polk et al., 2008; Wendell et al., 2010]. Full details of the dental exams have been previously described [Polk et al., 2008; Wang et al., 2010]. In brief, teeth were scored according to NIH/NIDCR-approved methods for assessing dental caries for research purposes via visual inspection, with each surface being classified as sound, precavitated, decayed (according to a four-level classification system), filled, missing due to decay, hypoplastic, or missing due to reasons other than decay, in accordance with the World Health Organization DMFS/ dfs scale. Two classes of tooth surfaces were defined: PFS included occlusal surfaces of molars and premolars, buccal surfaces of lower molars, and lingual surfaces of upper molars; SMS included all other tooth surfaces. Surface-level caries scores were used to generate the following four composite caries scores: (1) PFS DMFS score, which was the summation of decayed (D), missing due to decay (M), and filled (F) permanent dentition for PFS; (2) proportion PFS DMFS score, which was calculated by dividing the PFS DMFS score by the total number of permanent dentition PFS (i.e. surfaces present plus surfaces missing due to decay); (3) SMS $D M F S$ score, which was the summation of D, M, and F permanent dentition SMS; (4) proportion SMS DMFS, which was the SMS DMFS score divided by the number of permanent dentition SMS for which we have data. Four analogous caries scores were similarly calculated for primary tooth surfaces (i.e. PFS dfs, proportion PFS dfs, SMS dfs, and proportion SMS dfs), with the exception that the missing tooth component was not included in primary $\mathrm{dfs}$ scores due to measurement issues introduced by unerupted primary teeth. Likewise, composite variables were calculated for 'total' dentition (DMFS + dfs, etc.) by considering all teeth present without distinguishing between permanent and primary dentitions. Proportion caries scores were analyzed in order to account for the differences in number of teeth present among participants. Based on findings from previous genetic analyses in this sample [Wang et al., 2010], we have included pre-cavitated lesions (i.e. white spots) in the composite caries scores. Permanent and primary caries scores were analyzed for individuals based solely on the presence of teeth of each dentition type (i.e. not limited to specific age ranges). Third molars were excluded when calculating caries scores in this study. Endentulous family/household members, including infants, were excluded from the study $(n=60)$.

\section{Statistical Analysis}

The modeling framework used in this study estimates the heritability of a single phenotype and/or the genetic correlation between two phenotypes by comparing phenotype measurements across all pairs of relatives and nonrelatives while conditioning on the expected genetic sharing between pairs (e.g. that parents share $50 \%$ of their genetic material with their offspring, siblings share $50 \%$, half-siblings share $25 \%$, unrelated individuals share $0 \%$, etc.). Heritability estimates are interpreted as the portion of phenotype variation attributable to the cumulative effect of all genes affecting the phenotype. Genetic correlation estimates are interpreted as the degree to which two phenotypes are influenced by the same genetic effects.

Analyses were performed using variance decomposition methods, which partition the trait variance into environmental, heritable, and residual error components, while conditioning on the familial relationships among the study participants. This model takes the general form:

$$
y_{i}=\mu+\sum_{j}^{n} \beta_{j} x_{j i}+g_{i}+e_{i},
$$

where $y_{i}$ is the dental phenotype for the $\mathrm{i}$-th individual, $\mu$ is the sample mean, $X_{\mathrm{ji}}$ are the values of the predictors, $\mathrm{j}$, for the $\mathrm{i}$-th individual, $\beta_{j}$ are the regression coefficients for the $j$-th predictor, $g_{i}$ is the individual additive polygenic effect (based on expected genetic sharing due to the familial relationship between relative pairs), and $\mathrm{e}_{\mathrm{i}}$ is the individual residual error effect [Almasy and Blangero, 1998]. Pedigree-based likelihood methods were used to estimate model parameters, from which we calculated residual heritability $\left(h^{2}\right.$, i.e. the proportion of phenotype variance attributable to genetics after adjustment for covariates, age and sex). The statistical significance of heritability was assessed by the likelihood ratio test, comparing the model where the polygenic effect was estimated to the model where the polygenic effect was constrained to be zero. This test statistic asymptotically follows 50:50 mixture of the $\chi^{2}$ distribution with 1 degree of freedom and a point mass at zero.

A bivariate extension of the above univariate model was used to estimate the genetic correlation between caries scores, which describes the extent to which family structure explains the covariance of the two traits (i.e. whether the traits vary together through the family, based on expected genetic sharing among relatives). This model can loosely be interpreted as the extent to which the same or different genes affect the two traits. Likelihood ratio tests were used to assess the evidence for deviations of the genetic correlation from zero and 1 (i.e. whether genes similarly or differentially affect the two traits). Detailed explanation of the bivariate model is outside the scope of this article but is available elsewhere [Almasy et al., 1997]. All genetic analyses were performed using the Sequential Oligogenic Linkage Analysis Routines (SOLAR) software [Almasy and Blangero, 1998]. General statistics, data manipulations, and plotting of figures were performed in the $\mathrm{R}$ statistical suite ( $\mathrm{R}$ V2.8.1, R Foundation for Statistical Computing, Vienna, Austria).

The variance components methods described above assume normally distributed traits; however, caries scores exhibit skewed distributions. In general, variance components methods are known to be susceptible to large violations of normality [Allison et al., 1999]. Therefore, we addressed the possibility that model performance was adversely affected by deviations from normality by applying a severe normalization procedure to the data. First, caries scores were regressed on age and sex. Second, residuals were ranked and scaled (by dividing by the sample size +1 ) to range between zero and one. Finally, we applied the probit function (the quantile function or inverse cumulative distribution function for the standard normal distribution) to scaled rankings in order to generate exactly normally distributed traits. Analysis of transformed traits yielded strikingly similar results (not shown), suggesting that the skewed distributions of untransformed traits were not noticeably reducing model performance.

Overall, power to detect heritability of $25 \%$ or greater was high. Power was estimated empirically in SOLAR via 1,000 simulations of a normally distributed, heritable trait. Power to detect heritability of $h^{2}=0.25$ was $>99 \%$ for permanent caries scores, $81 \%$ for primary caries scores, and $>99 \%$ for total dentition caries scores. Power to detect heritability at $\mathrm{h}^{2}=0.30$ was near $100 \%$ for permanent, primary, and total dentition caries scores. 


\section{Results}

The demographic characteristics of the sample are shown in table 1. Prevalence of dental caries (i.e. the percentage of sample with one or more carious lesions) by age cohort is shown in table 2 . As expected, prevalence of dental caries was greater in the primary dentition than permanent dentition for children (age groups $<6$ years and 6-14 years). For all age groups, and for both primary and permanent dentitions, the prevalence of caries was greater (or equal) for PFS compared to SMS.

Furthermore, mean proportion caries scores were greater for PFS than SMS (data not shown). For example, the mean proportions of carious PFS and SMS were 0.32 and 0.09 , respectively, for permanent dentition, and 0.16 and 0.06 , respectively, for primary dentition. Note that proportion caries scores (i.e. the proportion of carious surfaces out of the total at-risk surfaces) takes into account the fact that the full dentition includes many more SMS than PFS.

Distributions of PFS and SMS caries scores (i.e. dfs, DMFS, and proportions thereof) are shown in figure 1. These distributions further exemplified the differences in caries experience in PFS compared to SMS. While fewer individuals experienced SMS caries, some of those who did exhibited many affected SMS. Due to the fact that many more SMS than PFS are at-risk for caries, the dfs and DMFS scores for SMS show a greater range of values (and greater variance), and contributed substantially to the total disease burden in the sample. This observation was especially evident for caries occurrence in the permanent dentition.

Table 3 shows the heritability for PFS and SMS caries, as well as the genetic correlations between them, for primary, permanent, and total (i.e. all teeth present regardless of dentition type) dentitions. In general, caries scores were moderately to highly heritable $\left(\mathrm{h}^{2}\right.$ estimates ranged from 17 to $53 \%$; $p<0.001$ for all phenotypes), indicating that the cumulative effect of genes accounted for a substantial portion of disease risk. Moreover the proportional caries scores exhibited greater heritability than the corresponding dfs and DMFS scores, suggesting that proportional caries scores, which take into account both the number of affected surfaces and number of at-risk surfaces, may be improved metrics for studying the genetics of dental caries.

Heritability of caries scores was generally similar between PFS and SMS (i.e. estimates were within 1-2 standard errors of each other). These results indicate that similar proportions of phenotype variation are explained by genes for PFS and SMS caries.

Dental Caries Genetics: Pit and Fissure and Smooth Surfaces
Table 1. Demographic characteristics of the study sample

\begin{tabular}{lc}
\hline Demographic characteristic & \\
\hline Sample size & 2,663 \\
Number of kinships & 740 \\
Mean size of kinships (range) & $4.72(1-20)$ \\
Number of relative pairs & \\
$\quad$ Parent-offspring & 1,736 \\
Siblings & 676 \\
Grandparent-grandchild & 60 \\
Half-siblings & 322 \\
Avuncular (i.e. uncle-nephew) & 124 \\
First cousins & 98 \\
Other relatives & 35 \\
Total related pairs & 3,051 \\
Within kinship unrelated pairs (i.e. spouses, etc.) & 739 \\
Total pairs & 3,790 \\
Self-reported whites, \% & 89.64 \\
Females, \% & 55.61 \\
Mean age, years (range) & 19.83 (1-93) \\
\hline
\end{tabular}

Genetic correlations between PFS and SMS caries scores were high $\left(\mathrm{rho}_{\mathrm{G}}\right.$ ranged from 0.76 to 1.00 ), which indicates that, for the most part, the role of genes is similarly acting on both types of tooth surfaces. In fact, for two of the phenotypes studied, model convergence occurred at the maximum possible value for the $\mathrm{rho}_{\mathrm{G}} \mathrm{pa}-$ rameter (i.e. 1.00), which is an unreliable point estimate, but nevertheless indicates high genetic correlation. Indeed, the high genetic correlation indicates a lack of evidence that separate genes are acting on PFS and SMS. Only for the dfs caries score in primary dentition was evidence observed for trait-specific genetic effects (i.e. $\left.\mathrm{rho}_{\mathrm{G}}<1.00, \mathrm{p}<0.001\right)$. All caries scores showed statistically significant evidence for common genetic effects jointly acting on PFS and SMS caries ( $\mathrm{p} \leq 0.001$ for all).

\section{Discussion}

We investigated the cumulative role of genes on caries scores in PFS and SMS and found that caries experience for both surface types was moderately to highly heritable, with proportional caries scores exhibiting higher heritability than dfs and DMFS scores. In addition to significant heritability, we showed that the genetic correlation between PFS and SMS caries scores was very high, indicating similar genetic effects acting on both surface types. Indeed, estimates for genetic correlation were not 
Table 2. Prevalence of dental caries as indicated by percent affected (number affected) by surface type: SMS caries only, PFS caries only, both SMS and PFS caries, and unaffected

\begin{tabular}{|c|c|c|c|c|c|c|c|c|c|c|}
\hline \multirow{2}{*}{$\begin{array}{l}\text { Age group, } \\
\text { years }\end{array}$} & \multicolumn{5}{|c|}{ Primary dentition caries } & \multicolumn{5}{|c|}{ Permanent dentition caries } \\
\hline & $\mathrm{n}$ & SMS only & PFS only & $\begin{array}{l}\text { both SMS } \\
\text { and PFS }\end{array}$ & unaffected & $\mathrm{n}$ & SMS only & PFS only & $\begin{array}{l}\text { both SMS } \\
\text { and PFS }\end{array}$ & unaffected \\
\hline$<6$ & 426 & $6 \%(25)$ & $6 \%(25)$ & $19 \%(82)$ & $69 \%(294)$ & 31 & $6 \%(2)$ & $13 \%(4)$ & $0 \%(0)$ & $81 \%(25)$ \\
\hline $6-14$ & 486 & $5 \%(22)$ & $14 \%(66)$ & $46 \%(224)$ & $36 \%(174)$ & 643 & $6 \%(39)$ & $25 \%(160)$ & $23 \%(150)$ & $46 \%(294)$ \\
\hline $14-18$ & - & - & - & - & - & 190 & $5 \%(10)$ & $26 \%(50)$ & $49 \%(93)$ & $19 \%(37)$ \\
\hline$>18$ & - & - & - & - & - & 1,044 & $2 \%(19)$ & $11 \%(114)$ & $84 \%(872)$ & $4 \%(39)$ \\
\hline
\end{tabular}

Fig. 1. Distributions of caries scores (i.e. dfs, DMFS) for PFS and SMS in primary and permanent dentitions. The histogram and corresponding left vertical axis indicates the number of individuals by caries score bin. The estimated cumulative density function (step plot) and corresponding right vertical axis shows the cumulative proportion of the sample having disease severity (from zero) up to a given caries score.

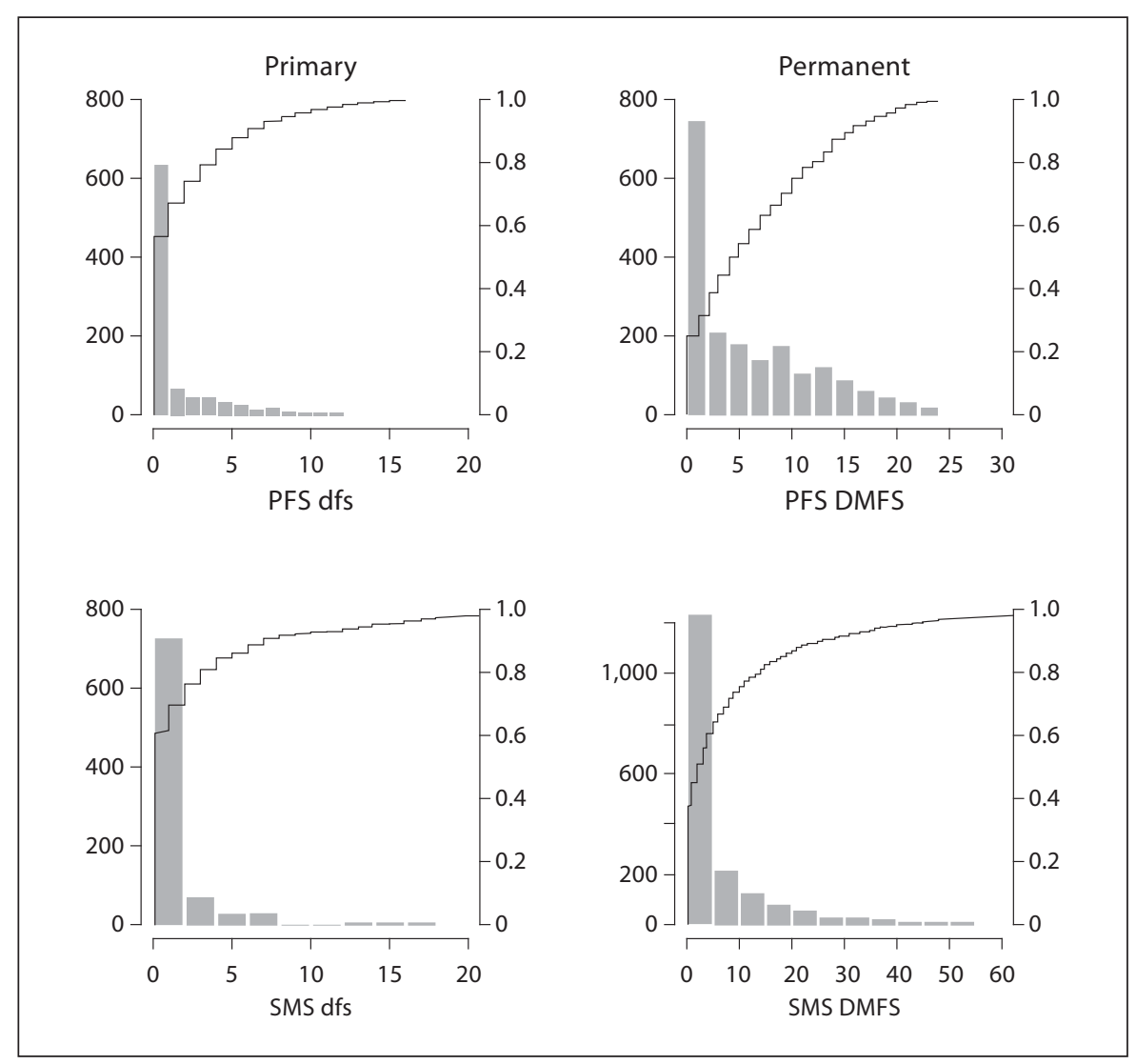

statistically different from 1.0 for DMFS, dfs + DMFS, proportion dfs, proportion DMFS, and proportion dfs + DMFS. In contrast, the genetic correlation between PFS and SMS for the dfs caries score was 0.76 , indicating that approximately $58 \%$ (i.e. rho $_{\mathrm{G}}{ }^{2}=0.76^{2}$ ) of the heritability of these two traits was explained by common genetic effects and approximately $42 \%$ by trait-specific genetic effects $(p<0.001)$. Trait-specific genetic effects may include genes that differentially impact dfs for PFS and
SMS in magnitude and/or direction. We caution that while genetic correlation was not statistically different from 1.0 for all other caries scores, our results indicated a lack of evidence for surface type-specific genetic effects, rather than evidence for a lack of surface type-specific genetic effects. In other words, true surface typespecific genetic effects may have gone undetected, but if so, they were likely small in comparison to common genetic effects. 
Table 3. Heritability and genetic correlation of PFS and SMS caries scores

\begin{tabular}{|c|c|c|c|c|c|c|c|c|c|c|c|c|c|}
\hline \multirow[t]{2}{*}{ Phenotype } & \multirow[t]{2}{*}{$\mathrm{n}$} & \multicolumn{4}{|l|}{ PFS } & \multicolumn{4}{|l|}{ SMS } & \multicolumn{4}{|c|}{ Shared genetics } \\
\hline & & $h^{2}$ & $\mathrm{SE}$ & $\mathrm{p}^{\mathrm{a}}$ & $\mathrm{R}^{2}$ & $h^{2}$ & SE & $\mathrm{p}^{\mathrm{b}}$ & $\mathrm{R}^{2}$ & $\mathrm{rho}_{\mathrm{G}}$ & $\mathrm{SE}$ & $\mathrm{p}_{\mathrm{rhoG}=0}{ }^{\mathrm{c}}$ & $\mathrm{p}_{\text {rhoG }=1}{ }^{\mathrm{d}}$ \\
\hline \multicolumn{14}{|l|}{ Primary dentition } \\
\hline $\mathrm{dfs}$ & 953 & 0.34 & 0.10 & $3 \times 10^{-4}$ & 0.01 & 0.42 & 0.10 & $2 \times 10^{-5}$ & 0.00 & 0.76 & 0.08 & 0.001 & $9 \times 10^{-4}$ \\
\hline Proportion dfs & 953 & 0.53 & 0.11 & $2 \times 10^{-6}$ & 0.04 & 0.42 & 0.10 & $2 \times 10^{-5}$ & 0.00 & 0.93 & 0.08 & $5 \times 10^{-6}$ & 0.208 \\
\hline \multicolumn{14}{|l|}{ Permanent dentition } \\
\hline DMFS & 1,939 & 0.27 & 0.06 & $7 \times 10^{-6}$ & 0.35 & 0.27 & 0.07 & $2 \times 10^{-4}$ & 0.22 & 1.00 & - & $2 \times 10^{-6}$ & 1.000 \\
\hline Proportion DMFS & 1,939 & 0.37 & 0.06 & $2 \times 10^{-11}$ & 0.33 & 0.21 & 0.06 & $2 \times 10^{-4}$ & 0.21 & 0.98 & 0.08 & $5 \times 10^{-9}$ & 0.424 \\
\hline \multicolumn{14}{|l|}{ Total dentition } \\
\hline $\mathrm{dfs}+\mathrm{DMFS}$ & 2,378 & 0.19 & 0.04 & $2 \times 10^{-6}$ & 0.28 & 0.17 & 0.04 & $1 \times 10^{-5}$ & 0.17 & 1.00 & - & $1 \times 10^{-7}$ & 1.000 \\
\hline Proportion dfs + DMFS & 2,378 & 0.31 & 0.04 & $2 \times 10^{-13}$ & 0.35 & 0.23 & 0.04 & $2 \times 10^{-8}$ & 0.19 & 0.99 & 0.06 & $6 \times 10^{-13}$ & 0.422 \\
\hline
\end{tabular}

$\mathrm{h}^{2}=$ Residual heritability after adjusting for sex and age; $\mathrm{SE}=$ standard error, not applicable for models that converged at rho $\mathrm{G}_{\mathrm{G}}=1.00$; $\mathrm{R}^{2}=$ proportion of trait variance attributable to sex and age; rho $_{\mathrm{G}}=$ genetic correlation, i.e. a measure of the extent to which traits covary due to genes.

${ }^{a} \mathrm{p}$ value that PFS caries is heritable. ${ }^{\mathrm{b}} \mathrm{p}$ value that SMS caries is heritable. ${ }^{\mathrm{c}} \mathrm{p}$ value that shared genetic risk factors affect both PFS and SMS. ${ }^{\mathrm{d}} \mathrm{p}$ value that unshared genetic risk factors differentially affect PFS and SMS.

Overall, the results of this report were consistent with a previous study in this sample on tooth-level caries scores (i.e. d1 ft, D1MFT, and d1ft + D1MFT in primary, permanent, and total dentitions, respectively) [Wang et al., 2010]. However, for all dentition types (i.e. primary, permanent, and total), heritability of both PFS and SMS were reduced compared to tooth-level caries scores. One explanation for the reduced heritability observed for PFS and SMS caries scores is that genetic factors may similarly affect caries risk for both surface types; therefore limiting analysis to one surface type may be less informative for the genetics of caries experience than simultaneous analysis of caries risk across both surface types. This notion is consistent with the high genetic correlation between PFS and SMS caries scores reported in this study.

While the current study did not seek to identify any specific genes affecting caries risk, it did show that PFS and SMS caries scores are heritable, and therefore may be useful phenotypes for future genetic association studies. The genetics of dental caries is thought to be very complex, with many biological mechanisms affecting cariogenesis and operating over long periods of time. Such mechanisms may include genes related to dietary choices (such as taste [Wendell et al., 2010] and olfactory receptors), immune response to pathogens [Bergandi et al., 2007], tooth enamel composition [Slayton et al., 2005], tooth morphology, saliva composition and flow rate, oral health behaviors, transmission of cariogenic bacteria among hosts [Law et al., 2007], and others. Genes acting through these and other avenues, some likely interacting with environmental factors, may each contribute only slightly to overall caries risk, and therefore teasing out the complex interplay of genetic and environmental risk factors represents a necessary challenge for better understanding of the multifactorial nature of dental caries.

Several limitations of the study warrant discussion. In particular, DMFS and dfs + DMFS caries scores for PFS and SMS were correlated, in part, due to the M surface classification. By convention, teeth missing due to decay contributed all surfaces to DMFS scores even though the actual carious lesion may have been limited to one surface type. Bias due to the M classification may have led to inflated estimation of the genetic correlation between PFS and SMS. In fact, the phenotypic correlation between PFS and SMS for the M component of DMFS was far greater than for D, F, or sound teeth (results not shown). However censoring the DMFS caries scores by removing the contribution of the M classification is also not ideal. Instead we suggest cautious interpretation of our genetic correlations given the known bias due to phenotype definitions. Likewise, restoration of approximal lesions is often performed via a two-surface filling, which results in the adjacent occlusal surface being counted toward PFS caries scores despite not necessarily having been decayed. This may have caused inflated PFS caries scores. Again, such bias may lead to inflated estimation of genetic correlation. 
Another limitation of this study was that all tooth surfaces were dichotomized as either PFS or SMS, which may not fully reflect the complex hierarchy of surface-specific cariological risk factors. The epidemiology of surfacespecific caries incidence suggests differences in caries susceptibility (and therefore suggests differences in caries risk factors) among PFS and among SMS [Batchelor and Sheiham, 2004; Psoter et al., 2009]. Future development of improved systems for categorizing and analyzing tooth surfaces may assist in discovering the surface-level factors leading to disease. For example, further subdivisions of surface types such as approximal versus buccal/lingual SMS, mandibular vs. maxillary, etc., may also be important for assessing the differential effects of environmental and genetic risk factors. Additionally, risk modification by age and, in particular, the duration of time that surfaces were present (i.e. at risk) is difficult to model for primary, permanent, and combined dentitions. Moreover, differences in environmental factors, such as fluoride varnish and sealants, were not modeled in these analyses, but may have differentially impacted caries scores among study participants leading to noise in the phenotype assessments. Indeed, other studies have shown important differential effects of fluoride exposure, dietary habits, socioeconomic status, and age on PFS and SMS caries [Maupome et al., 2001; Jiang et al., 2005; Warren et al., 2006].

Lastly, our method of data collection - visual inspection with dental explorer - is itself limited in that it is not the gold standard method for clinical caries assessment and may lead to deflated caries scores. However, assessment of data quality in our sample [Polk et al., 2008; Wendell et al., 2010] has shown that our method produces reliable and reproducible data of sufficient quality for pursuing the goals of this study. Moreover, our methods of data analysis are very robust. In general, measurement error or bias, model misspecification, and/or any unknown factors introducing noise into the phenotype would not lead to false positive findings (because measurement issues would not affect data proportional to biological relatedness among participants), but would instead bias these analyses toward the null hypothesis. Therefore, despite these limitations, the analyses are likely conservative and the general conclusions from this study are robust.

This study benefits from many strengths, including a large sample from an understudied, high-risk population. Our household-based recruitment yielded a rich sample comprised of a variety of relative types, which greatly improved our estimation of heritability and ge- netic correlation compared to studies limited to one or few relative types (i.e. studies of siblings, parent-offspring trios, etc.). This is because extended relatives (especially relatives residing in separate households such as half-siblings, cousins, etc.) are less likely to share familial nongenetic factors that could lead to overestimation. Our study was adequately powered, and all significant $p$ values far exceeded the significance thresholds for Bonferroni adjustment for multiple comparisons. Additionally, we assessed the effect of nonnormality of our trait distributions by repeating analysis using exactly normal transformed phenotypes. Heritability and genetic correlation estimates were nearly identical, suggesting that deviations of caries scores from normality did not adversely affect our modeling framework.

The major conclusion from this study was that caries scores were heritable and that the majority of genes affecting caries risk were common to both PFS and SMS. While some surface type-specific genetic effects may exist, especially for dfs scores in primary dentition, the high genetic correlations suggest that combining PFS and SMS in future efforts to identify genes involved in caries risk may be beneficial complement to studying PFS and SMS separately. Additionally, this study highlighted the need for better caries phenotypes and/or modeling approaches that more accurately capture the complexity of the distribution of caries risk across the dentition. Additional work is currently needed to identify the environmental and genetic factors that differentially affect caries risk across surface types, and to devise better categorization methods that sensibly group tooth surfaces based on common risk profiles. This study is one of few attempts at defining new traits and genetic models which may assist in finding the specific genes implicated in caries etiology, and lead to improved understanding, and prevention, of the factors leading to disease.

\section{Acknowledgments}

We would like to extend our sincere thanks to the participants of this study for their contribution toward our goals of understanding and bettering the oral health of rural Appalachian communities. We would also like to acknowledge the contributions of our field staff for their data collection efforts. The following community health care organizations participated in this study: University of Pittsburgh Center for Rural Health Practice, Bradford, Pa.; McKean County Dental Center, Bradford, Pa.; Cornerstone Care Community Medical and Dental Center, Burgettstown, Pa.; UPMC Braddock Hospital, Braddock, Pa.; Camden-on-Gauley Medical Center, Camden-on-Gauley, W.Va.; Community Health Clinic of Nicholas County, Summersville, W.Va.; Richwood Area 
Community Hospital, Richwood, W.Va.; Summersville Memorial Hospital, Summersville, W.Va.; Webster County Memorial Hospital, Webster Springs, W.Va. Additional thanks is given to the GORGE Connection Rural Health Education Partnership Board, the Webster-Nicholas Rural Health Education Consortium Board, the West Virginia Rural Health Education Partnerships program, the Nicholas and Webster Boards of Education, the UPMC Braddock Community Advisory Board, and the individuals and social service agencies that helped develop the Center for Oral Health Research in Appalachia. Lastly, we would like to acknowledge three anonymous reviewers for their thoughtful feedback and commentary on this work.

Support for this study was provided by the National Institute of Dental and Craniofacial Research, including grants R03DE021425, R01-DE014899, and U01-DE018903, as part of the NIH Genes and Environment Initiative (GENEVA), and by U01HG004446 for data cleaning by the GENEVA Coordinating Center. Genotyping was provided by the NIDCR through a federal contract from the National Institutes of Health to The Johns Hopkins University, Center for Inherited Disease Research, NIH contract No. HHSN268200782096C. Additional support was provid- ed by the University of Pittsburgh School of Dental Medicine, the West Virginia University School of Dentistry and Eberly College of Arts and Sciences. The content presented herein is solely the responsibility of the authors and does not necessarily represent the official views of the National Institute of Dental and Craniofacial Research, nor the National Institutes of Health.

The funders had no role in study design, data collection and analysis, decision to publish, or preparation of the manuscript. Co-author contributions are as follows: conceived and designed the COHRA initiative: R.J.W., R.C., D.W.N., M.L.M.; conceived and designed this study: J.R.S., X.W., R.J.W., K.T.C., M.L.M.; analyzed the data: J.R.S., X.W.; interpreted the results: J.R.S., X.W., R.S.D., S.W., R.J.W., K.T.C., R.C., D.W.M., M.L.M.; wrote the paper: J.R.S., X.W., R.J.W., K.T.C., M.L.M.

\section{Disclosure Statement}

All co-authors have no conflicts of interest.

\section{References}

- Allison DB, Neale MC, Zannolli R, Schork NJ, Bretz WA, Corby PM, Schork NJ, Robinson MT, Amos CI, Blangero J: Testing the robustness of the likelihood-ratio test in a variancecomponent quantitative-trait loci-mapping procedure. Am J Hum Genet 1999;65:531544.

Almasy L, Blangero J: Multipoint quantitativetrait linkage analysis in general pedigrees. Am J Hum Genet 1998;62:1198-1211.

-Almasy L, Dyer TD, Blangero J: Bivariate quantitative trait linkage analysis: pleiotropy versus co-incident linkages. Genet Epidemiol 1997; 14:953-958.

-Anderson M: Risk assessment and epidemiology of dental caries: review of the literature. Pediatr Dent 2002;24:377-385.

Batchelor PA, Sheiham A: Grouping of tooth surfaces by susceptibility to caries: a study in 5-16 year-old children. BMC Oral Health 2004;4:2.

-Bergandi L, Defabianis P, Re F, Preti G, Aldieri E, Garetto S, Bosia A, Ghigo D: Absence of soluble cd14 in saliva of young patients with dental caries. Eur J Oral Sci 2007;115:93-96.

Boraas JC, Messer LB, Till MJ: A genetic contribution to dental caries, occlusion, and morphology as demonstrated by twins reared apart. J Dent Res 1988;67:1150-1155.

Bretz WA, Corby PM, Hart TC, Costa S, Coelho MQ, Weyant RJ, Robinson M, Schork NJ: Dental caries and microbial acid production in twins. Caries Res 2005a;39:168-172.

Bretz WA, Corby PM, Melo MR, Coelho MQ, Costa SM, Robinson M, Schork NJ, Drewnowski A, Hart TC: Heritability estimates for dental caries and sucrose sweetness preference. Arch Oral Biol 2006;51:1156-1160. Coelho M, Costa S, Melo Filho MR, Weyant RJ, Hart TC: Longitudinal analysis of heritability for dental caries traits. J Dent Res 2005b;84:1047-1051.

Cornelis MC, Agrawal A, Cole JW, Hansel NN, Barnes KC, Beaty TH, Bennett SN, Bierut LJ, Boerwinkle E, Doheny KF, Feenstra B, Feingold E, Fornage M, Haiman CA, Harris EL, Hayes MG, Heit JA, Hu FB, Kang JH, Laurie CC, Ling $\mathrm{H}$, Manolio TA, Marazita ML, Mathias RA, Mirel DB, Paschall J, Pasquale LR, Pugh EW, Rice JP, Udren J, van Dam RM, Wang X, Wiggs JL, Williams K, Yu K: The Gene, Environment Association Studies consortium (GENEVA): maximizing the knowledge obtained from GWAS by collaboration across studies of multiple conditions. Genet Epidemiol 2010;34:364-372.

From the Centers for Disease Control and Prevention: Total tooth loss among persons aged $>$ or $=65$ years - selected states, 1995-1997. JAMA 1999;281:1264-1266.

Goodman HO, Luke JE, Rosen S, Hackel E: Heritability in dental caries, certain oral microflora and salivary components. Am J Hum Genet 1959;11:263-273.

-Grembowski D, Conrad DA, Milgrom P: Dental care demand among children with dental insurance. Health Serv Res 1987;21:755-775.

Hunter PB: Risk factors in dental caries. Int Dent J 1988;38:211-217.
-Janes GR, Blackman DK, Bolen JC, Kamimoto LA, Rhodes L, Caplan LS, Nadel MR, Tomar SL, Lando JF, Greby SM, Singleton JA, Strikas RA, Wooten KG: Surveillance for use of preventive health-care services by older adults, 1995-1997. MMWR CDC Surveill Summ 1999;48:51-88.

- Jiang H, Tai B, Du M, Peng B: Effect of professional application of APF foam on caries reduction in permanent first molars in 6-7-year-old children: 24-month clinical trial. J Dent 2005;33:469-473.

Laurie CC, Doheny KF, Mirel DB, Pugh EW, Bierut LJ, Bhangale T, Boehm F, Caporaso NE, Cornelis MC, Edenberg HJ, Gabriel SB, Harris EL, Hu FB, Jacobs KB, Kraft P, Landi MT, Lumley T, Manolio TA, McHugh C, Painter I, Paschall J, Rice JP, Rice KM, Zheng X, Weir BS: Quality control and quality assurance in genotypic data for genome-wide association studies. Genet Epidemiol 2010;34:591-602.

Law V, Seow WK, Townsend G: Factors influencing oral colonization of mutans streptococci in young children. Aust Dent J 2007;52:93100; quiz 159 .

Liu H, Deng H, Cao CF, Ono H: Genetic analysis of dental traits in 82 pairs of female-female twins. Chin J Dent Res 1998;1:12-16.

Marosy B, Romm J, Hetrick K, Doheny K, Pugh E, Tsai Y: Development of a low cost SNP barcode panel (abstract 2653f). Am Soc Hum Genet 57th Annu Meet, San Diego, 2007.

-Maupome G, Shulman JD, Clark DC, Levy SM, Berkowitz J: Tooth-surface progression and reversal changes in fluoridated and no-longer-fluoridated communities over a 3-year period. Caries Res 2001;35:95-105. 
-Mobley C, Marshall TA, Milgrom P, Coldwell SE: The contribution of dietary factors to dental caries and disparities in caries. Acad Pediatr 2009;9:410-414.

O'Connell JR, Weeks DE: PedCheck: a program for identification of genotype incompatibilities in linkage analysis. Am J Hum Genet 1998;63:259-266.

Polk DE, Weyant RJ, Crout RJ, McNeil DW, Tarter RE, Thomas JG, Marazita ML: Study protocol of the Center for Oral Health Research in Appalachia (COHRA) etiology study. BMC Oral Health 2008;8:18.

-Psoter WJ, Pendrys DG, Morse DE, Zhang HP, Mayne ST: Caries patterns in the primary dentition: cluster analysis of a sample of 5,169 Arizona children 5-59 months of age. Int J Oral Sci 2009;1:189-195.
Public Health and Aging: Retention of natural teeth among older adults - United States, 2002. MMWR Morb Mortal Wkly Rep 2003; 52:1226-1229.

Purnell LD, Counts M: Appalachians; in Purnell LD, Paulanka BJ (eds): Transcultural Health Care: A Culturally Competent Approach. Philadelphia, Davis, 1998.

Shuler CF: Inherited risks for susceptibility to dental caries. J Dent Educ 2001;65:10381045.

Slayton RL, Cooper ME, Marazita ML: Tuftelin, mutans streptococci, and dental caries susceptibility. J Dent Res 2005;84:711-714.
Vieira AR, Marazita ML, Goldstein-McHenry T: Genome-wide scan finds suggestive caries loci. J Dent Res 2008;87:435-439.

-Wang X, Shaffer JR, Weyant RJ, Cuenco KT, DeSensi RS, Crout R, McNeil DW, Marazita ML: Genes and their effects on dental caries may differ between primary and permanent dentitions. Caries Res 2010;44:277-284.

Warren JJ, Levy SM, Broffitt B, Kanellis MJ: Longitudinal study of non-cavitated carious lesion progression in the primary dentition. J Public Health Dent 2006;66:83-87.

-Wendell S, Wang X, Brown M, Cooper ME, DeSensi RS, Weyant RJ, Crout R, McNeil DW, Marazita ML: Taste genes associated with dental caries. J Dent Res 2010;89:11981202. 